\title{
Développement d'un Guide de prévention, évaluation et traitement des endommagements des réseaux en polyéthylène pour la distribution du gaz
}

\author{
Dominique Gueugnaut ${ }^{1, a}$, Alain Darut ${ }^{2}$ et Jean-Claude Bergeron ${ }^{3}$ \\ ${ }^{1}$ Gaz de France, Direction de la Recherche, Département Réseaux, 93211 Saint-Denis La Plaine Cedex, France \\ 2 EDF Gaz de France Distribution, 92050 Paris-La Défense Cedex, France \\ 3 Gaz de France, Service de la Formation Professionnelle, Pôle Technique Gaz, 92635 Gennevilliers Cedex, France
}

Reçu le 28 mai 2003, accepté le 9 janvier 2004

\begin{abstract}
Résumé - Début 2002, le réseau de distribution de Gaz de France représentait plus de $155000 \mathrm{~km}$ de canalisations dont plus de $95000 \mathrm{~km}$ étaient constitués de polyéthylène, cette part étant en constante augmentation par rapport aux matériaux métalliques traditionnels. Même si les canalisations les plus anciennes ont été posées au milieu des années 70, le retour d'expérience montre une excellente tenue dans le temps du polyéthylène, dans des conditions normales de mise en œuvre et d'exploitation. Dans un souci constant d'amélioration de la qualité et de la sécurité de la chaîne des ouvrages gaz en polyéthylène, Gaz de France a mis en place un système d'assurance qualité devant permettre d'éviter toute dérive dans la réalisation de ses réseaux, depuis la fabrication des résines, tubes et accessoires, jusqu'à l'exploitation des canalisations sous 4 ou 8 bar de pression. Ce système d'assurance qualité repose sur un ensemble d'outils et de méthodologies qui se mettent en place progressivement. Le Guide de prévention, évaluation et traitement des endommagements, qui constitue l'un de ces outils, a été élaboré fin 1999 dans le but de mettre à disposition un ouvrage de référence destiné à sensibiliser la profession aux risques et aux conséquences des endommagements subis par les canalisations PE dans des conditions de fabrication, de stockage, de manutention, de pose ou d'exploitation incorrectement maîtrisées.
\end{abstract}

Mots clés : Polyéthylène $(\mathrm{PE})$ / réseaux de distribution de gaz / endommagements / prévention / durée de vie / sûreté / recommandations / fiabilité

\begin{abstract}
Development of Guidelines for Prevention, Evaluation and Treatment of damage to polyethylene pipelines. Beginning of 2002 the Gaz de France distribution network was composed of more than $155000 \mathrm{~km}$ of pipeline of which $95000 \mathrm{~km}$ were made of polyethylene, a share that is increasing compared with traditional metallic materials. Even if the oldest sections of pipe were laid in the midseventies, experience has shown that polyethylene shows good lasting properties under normal installation and operating conditions. In its quest for continuous improvement in the quality and safety of the chain of gas installations in polyethylene, Gaz de France has established a quality assurance system that should eliminate any deviation from established standards in the construction of its networks. This covers the entire process chain, from the manufacture of the resins, pipe and fittings to the operation of the pipelines under 4 to 8 bars of pressure. This quality assurance system is based on a set of tools and methodologies that is progressively being established. The Damage Prevention, Evaluation and Treatment Guide, one of these tools, was prepared at the end of 1999 with the objective of making a work of reference available that draws the attention of workers in the profession to the risks and consequences of damage that might occur to PE piping under incorrectly controlled conditions of manufacture, storage, maintenance, laying and operation.
\end{abstract}

Key words: Polyethylene (PE) / gas distribution networks / damage prevention / lifespan / safety / guidelines / reliability

\footnotetext{
a Auteur correspondant :

dominique.gueugnaut@gazdefrance.com
} 


\section{Introduction}

Début 2002, le réseau de distribution de Gaz de France représentait plus de $155000 \mathrm{~km}$ de canalisations dont plus de $95000 \mathrm{~km}$ étaient constitués de polyéthylène, cette part étant en constante augmentation par rapport aux matériaux métalliques traditionnels.

Même si les canalisations les plus anciennes ont été posées au milieu des années 70 , le nombre d'incidents avec fuites sur le réseau demeure à un niveau extrêmement faible, dont la grande majorité est « piégée » grâce aux procédures de surveillance mises en œuvre, dont l'emploi est de plus en plus généralisé.

Dans ces conditions, même si la garantie d'une bonne tenue dans le temps du réseau semble a priori acquise, comme l'indique le retour d'expérience sur bientôt 30 ans ou plus en France ou dans le monde entier, Gaz de France souhaite disposer d'outils et de méthodes permettant de suivre en temps réel l'état de ses réseaux et de contrôler leur évolution dans le temps, afin d'être en mesure d'anticiper les incidents susceptibles de se produire et de déterminer les actes d'exploitation pour y faire face, sur la base de critères scientifiquement établis (zones à risques, critères d'endommagement).

Dans ce cadre, Gaz de France maintient des efforts de recherche significatifs et mène des études de fond au travers de projets pris en charge par sa Direction de Recherche.

En parallèle, dans un souci constant d'amélioration de la qualité de la chaîne des ouvrages gaz en polyéthylène, Gaz de France a mis en place un système d'assurance qualité devant permettre d'éviter toute dérive dans la réalisation de ses réseaux, depuis la fabrication des résines, tubes et accessoires, jusqu'à l'exploitation des canalisations sous 4 voire 8 bar de pression (la réglementation actuelle limitant à 10 bar la pression d'utilisation des canalisations de distribution de gaz naturel).

Ce système d'assurance qualité repose sur un ensemble d'outils et de méthodologies déjà en place ou en passe de l'être, tant au niveau de la fabrication $[1,2]$ - qui passe notamment par la certification des fabricants (ISO, marque NF) - que de la réalisation des ouvrages - qui passe notamment par la formation et la qualification des opérateurs - ou encore de l'exploitation de ces ouvrages dont l'organisation du retour d'expérience constitue un axe majeur.

Un système complet de traçabilité des tubes et accessoires viendra compléter à très court terme cette chaîne d'assurance qualité, en parfaite harmonie avec le standard ISO paru en novembre 2003 [3-7].

En termes de réalisation des réseaux $\mathrm{PE}$ gaz (mise en ouvre), outre la formation et la qualification des opérateurs de soudage, des ouvrages à caractère pédagogique ont été élaborés par Gaz de France.

Parmi ces ouvrages, un guide dit de l'électrosoudage est disponible depuis le début des années 90 [8]. Ce guide présente les procédures qui permettent de réaliser les soudures par électrofusion des tubes et accessoires PE de manière correcte et qui garantissent de manière connexe la tenue des assemblages sur le long terme.
Le Guide de prévention, évaluation et traitement des endommagements, qui fait l'objet de cet article, a été élaboré pour sa part fin 1999 [9]. La vocation de ce guide est de mettre à disposition du public un ouvrage de référence destiné à sensibiliser la profession aux risques et aux conséquences des endommagements subis par les canalisations PE dans des conditions de fabrication, de stockage, de manutention, de pose ou d'exploitation incorrectement maîtrisées.

Le guide est associé à des méthodes de visualisation, de caractérisation et de réparation des endommagements sur canalisations $\mathrm{PE}$, qui permettent de vérifier in situ les seuils admissibles en matière d'endommagement répertoriés dans le guide, et de pallier le cas échéant aux éventuels problèmes rencontrés.

\section{Architecture du Guide de prévention, évaluation et traitement des endommagements}

Ce guide «Grand Public» se veut un ouvrage de référence à destination des entreprises prestataires d'EDF Gaz de France Distribution pour la pose des canalisations en polyéthylène. Il est complémentaire à la fois du guide de l'électrosoudage et du guide de la Distribution (à usage interne d'EDF Gaz de France Distribution).

Le Guide de prévention, évaluation et traitement des endommagements se présente sous forme d'un livret broché au format A5, comportant 85 pages couleurs recto-verso revêtues d'une plastification adaptée pour une utilisation sur le terrain.

Une plastification adéquate du document permet de l'utiliser dans les conditions parfois difficiles du terrain (pluie, boue, ....).

L'ouvrage est constitué de quatre parties distinctes.

\subsection{Partie I : règles générales de mise en œuvre pour prévenir les endommagements}

La partie I définit les règles générales de mise en œuvre pour éviter les endommagements sur tubes et accessoires lors du stockage, du transport et de la manutention, ainsi que dans différentes configurations de pose (tranchée ouverte, tubage, forage dirigé, pose à proximité d'un réseau de chaleur).

Cette partie, constituée de sept sections distinctes, est construite de manière symétrique avec, en regard, les risques encourus et les recommandations pour éviter ces risques. Les sept sections concernent le stockage, le transport et la manutention, la mise en œuvre des tourets et des couronnes, la pose en tranchée, le tubage, le forage dirigé, et enfin, la pose à proximité des réseaux de chaleur et des câbles électriques.

Pour ce qui concerne la phase de stockage des tubes et accessoires notamment, le guide rappelle les conditions optimales à respecter - tant en termes d'environnement qu'en termes de durée maximale admissible d'exposition 


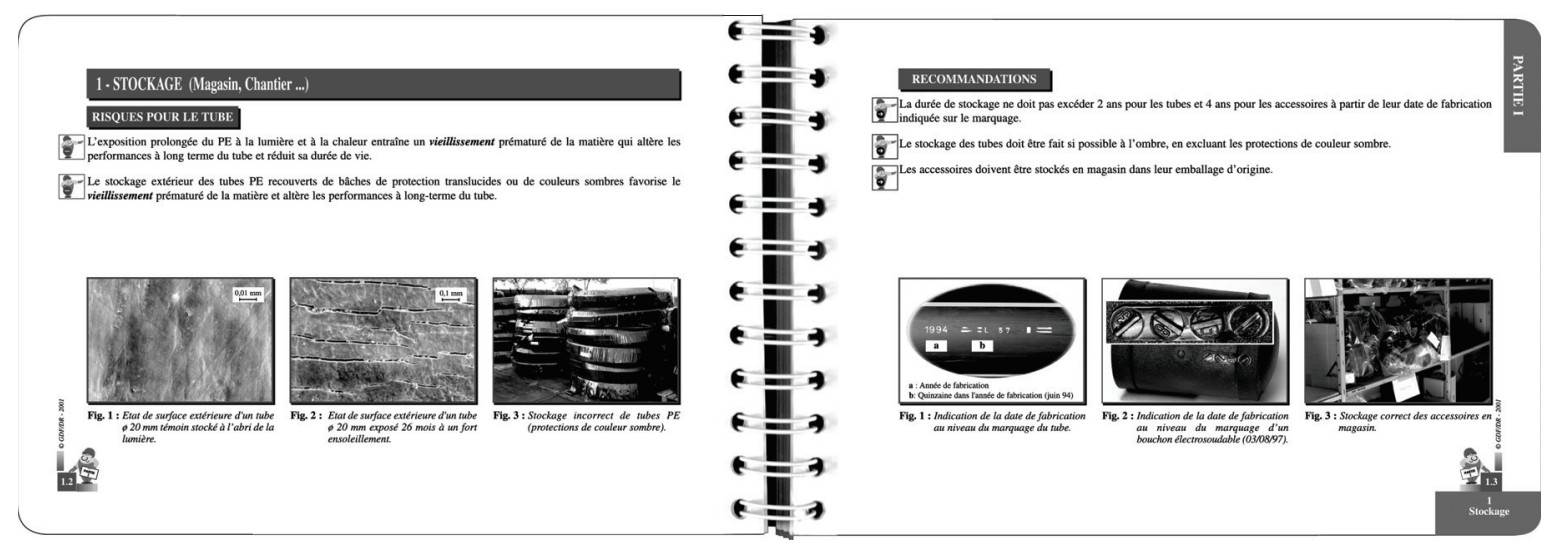

Fig. 1. Risques et recommandations liés au stockage des tubes PE (partie I du guide).

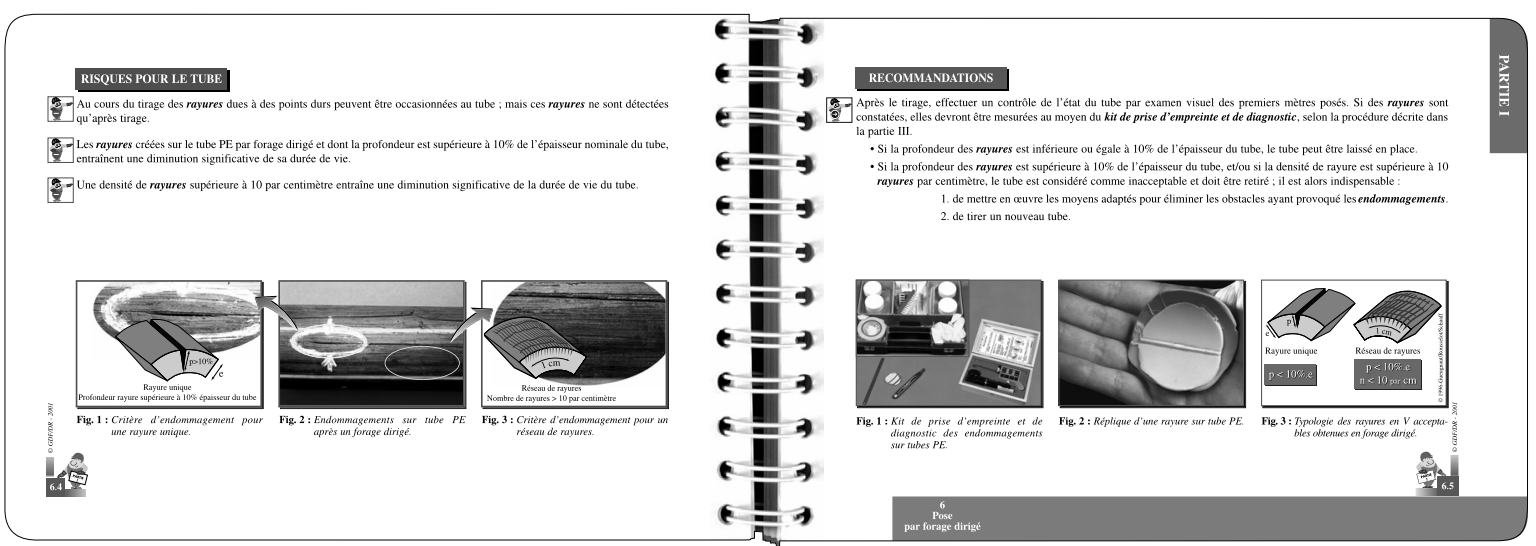

Fig. 2. Risques et recommandations liés à la pose par forage dirigé des tubes PE (partie I du guide).

- pour éviter l'occurrence d'une dégradation photothermo-oxydante dont les conséquences sont l'apparition de microfissures de surface susceptibles de dégénérer ensuite dans la masse par propagation lente de fissure (Fig. 1).

Pour ce qui concerne par exemple la pose par forage dirigé, le guide rappelle les conséquences de la création de rayures ou d'entailles en surface extérieure du tube, consécutive à une opération de tubage incorrecte, et présente les moyens de mesure associés à ces défauts ainsi que le seuil maximal admissible en termes de profondeur de la rayure (Fig. 2).

Pour ce qui concerne la pose à proximité de sources de chaleur ou de câbles électriques (ex. réseaux de chauffage urbains) le guide rappelle les risques encourus pour le tube tant à court terme (ex. fusion localisée avec fuite immédiate), qu'à moyen terme (ex. gonflement sous l'effet du ramollissement du matériau, propagation lente thermo-activée d'une fissure à partir d'un défaut préexistant) et définit les distances interconcessionnaires minimales à respecter dans le cas où le tube PE est dépourvu de protection.

Dans le cas où ces distances minimales ne peuvent être respectées, le guide définit également les caractéristiques des isolants thermomécaniques dont le tube PE devra être revêtu au moment de la pose (Fig. 3).

\subsection{Partie II : Nocivité des endommagements}

La partie II présente les relations de cause à effet entre différentes configurations potentiellement endommageantes pour le PE (ex. appui sur point dur, présence d'entailles extérieures, proximité de sources de chaleur, écrasement, flexion excessive, ... ) et sa durée de vie.

Cette partie est constituée de 9 sections correspondant à des configurations dites « génériques » en termes de représentativité par rapport au terrain Les neuf sections concernent : le comportement rhéologique particulier du $\mathrm{PE}$, les phénomènes d'oxydation provoqués par les UV et la chaleur, les phénomènes de fissuration liés à la présence d'entailles ou de défauts, les phénomènes de fragilisation à basse température, l'effet des agents tensio-actifs, l'effet des condensats et des produits chimiques, l'effet de l'écrasement, les effets d'un cintrage ou d'une flexion excessifs, les effets d'une traction excessive et enfin, les effets des poinçonnements sur points durs.

Les informations contenues dans ces sections expliquent de manière très synthétique le cheminement suivi pour valider les seuils d'endommagement indiqués dans la partie I du Guide.

Les deux exemples qui suivent illustrent la démarche suivie pour établir les critères de nocivité des endommagements de type oxydation et fissuration sur entaille pré-existante. 


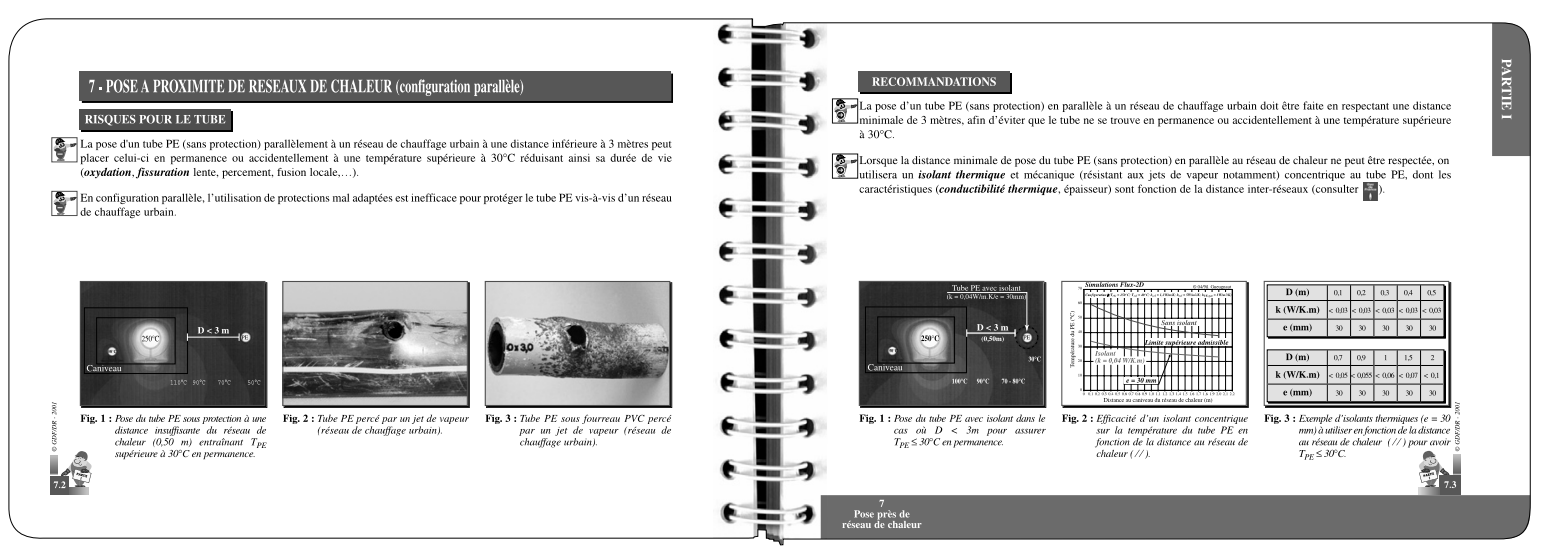

Fig. 3. Risques et recommandations liés à la pose à proximité d'un réseau de chaleur ou de câbles électriques (partie I du guide).
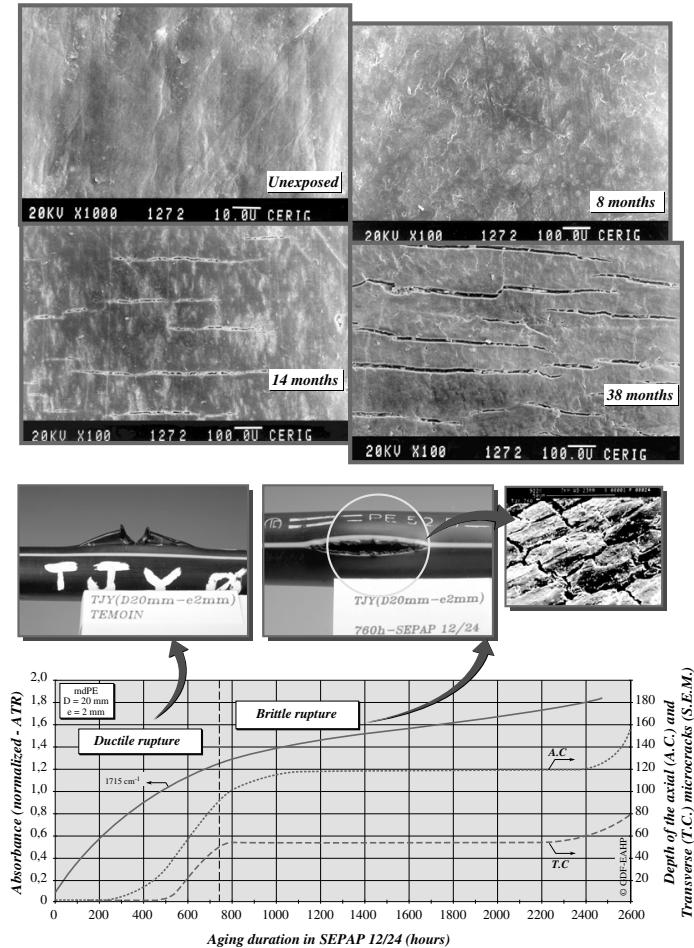

Fig. 4. Micro fissuration en surface extérieure d'un tube PE sous l'effet des conditions climatiques naturelles et influence sur le comportement en pression instantané.

\subsubsection{Phénomènes d'oxydation}

Comme toute polyoléfine, le PE donne lieu sous l'effet de la lumière et de la chaleur à des phénomènes de photoet thermo-oxydation qui conduisent inévitablement à plus ou moins long terme - malgré la présence de stabilisants dans la matrice - au vieillissement prématuré du matériau et à une modification connexe - généralement défavorable - de ses caractéristiques chimiques, physiques et mécaniques [10-18] (Fig. 4).

Ce phénomène est prépondérant lors d'une exposition climatique prolongée comme par exemple lors de la phase de stockage des tubes en extérieur mais également
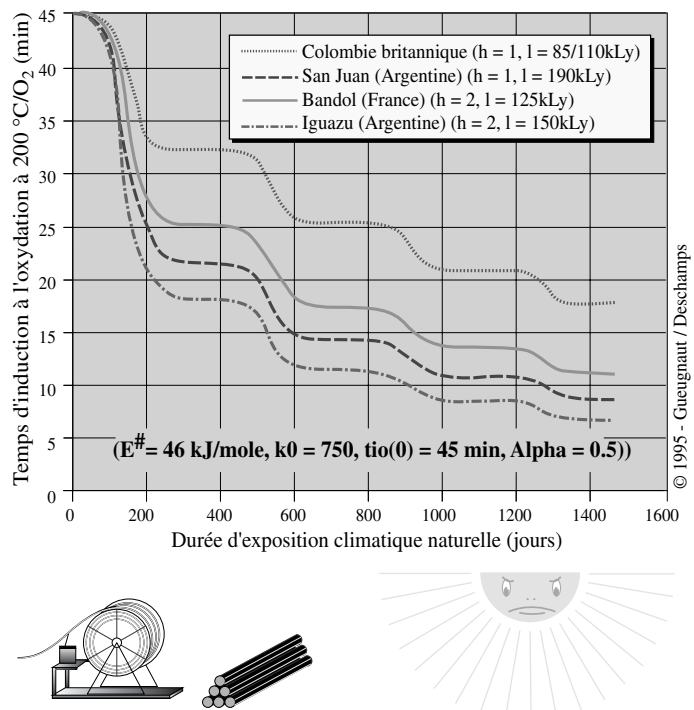

\begin{tabular}{|c|c|c|c|c|c|c|}
\hline SIMUSTAB $^{\circledR}$ & Bandol & Paris & Lille & Toulouse & Brest & Strasbourg \\
\hline $\begin{array}{c}\text { Stockage en été } \\
(07)\end{array}$ & $\begin{array}{c}3224 \text { jours } \\
(8,8 \text { ans })\end{array}$ & $\begin{array}{c}4015 \text { jours } \\
(11 \text { ans })\end{array}$ & $\begin{array}{c}6144 \text { jours } \\
(16,8 \text { ans })\end{array}$ & $\begin{array}{c}3650 \text { jours } \\
(10 \text { ans })\end{array}$ & $\begin{array}{c}5444 \text { jours } \\
(14,9 \text { ans })\end{array}$ & $\begin{array}{c}5110 \text { jours } \\
(14 \text { ans })\end{array}$ \\
\hline $\begin{array}{c}\text { Stockage en hiver } \\
\text { (12) }\end{array}$ & $\begin{array}{c}3133 \text { jours } \\
(8,6 \text { ans })\end{array}$ & $\begin{array}{c}4228 \text { jours } \\
(11,- \text { ans })\end{array}$ & $\begin{array}{c}6022 \text { jours } \\
(16,5 \text { ans })\end{array}$ & $\begin{array}{c}3741 \text { jours } \\
(10,2 \text { ans })\end{array}$ & $\begin{array}{c}5323 \text { jours } \\
(14,6 \text { ans })\end{array}$ & $\begin{array}{c}5262 \text { jours } \\
(14,4 \text { ans })\end{array}$ \\
\hline
\end{tabular}

Fig. 5. Diminution simulée de la stabilité chimique en surface extérieure d'un tube PE exposée sous différents climats représentatifs et durées critiques d'exposition climatiques naturelles pour différents sites d'exposition français.

lors d'une exposition prolongée à une source de chaleur, comme par exemple à proximité d'un réseau de chauffage urbain opérant dans certaines conditions défavorables (défauts d'isolation, réseau « chaud $», \ldots$ ).

Le logiciel SIMUSTAB ${ }^{\circledR}$ permet de calculer les durées maximales d'exposition d'un tube PE avant microfissuration critique de sa surface extérieure à partir du suivi d'un indicateur représentatif de l'avancée de l'oxydation de la matière en surface extérieure, en prenant en compte à la fois la nature du PE et de son système stabilisant ainsi que les conditions de l'environnement et leurs fluctuations (température, fraction UV) (Fig. 5). 

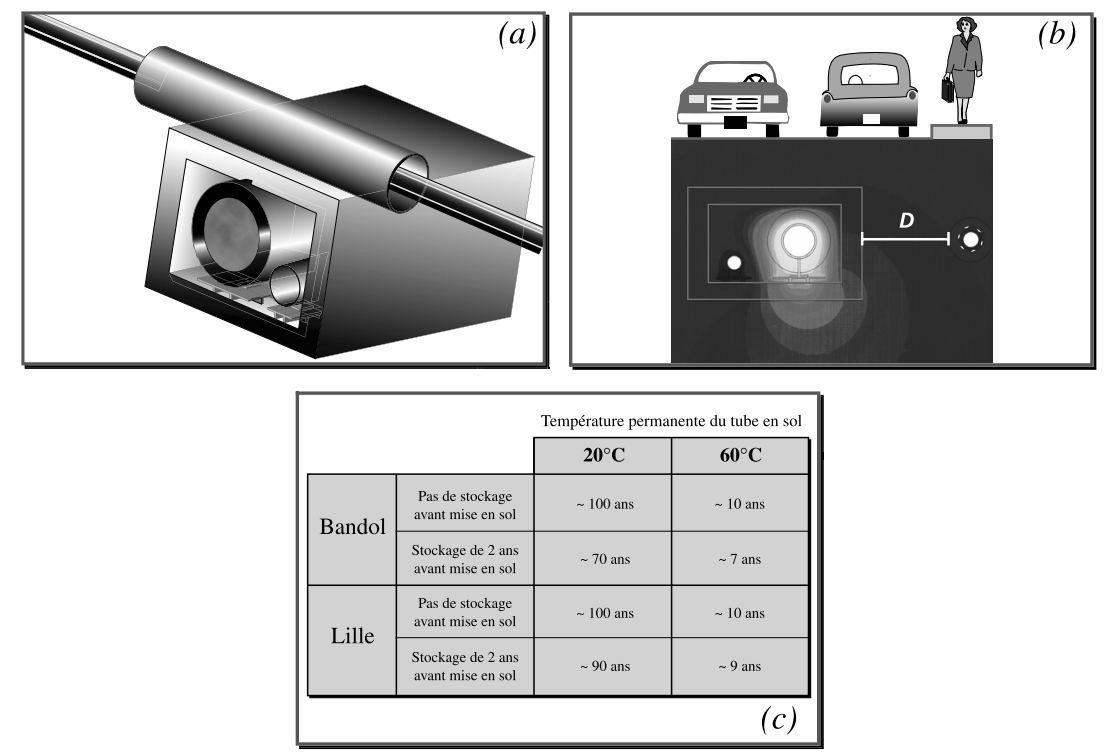

Fig. 6. Configurations de pose d'un tube PE à proximité d'un réseau de chaleur (a, b) et durée de maintien critique avant endommagement du tube en fonction de ses conditions de stockage et de sa température permanente en sol (c).

Les résultats numériques, calés sur les résultats expérimentaux obtenus sur le site de Bandol ainsi qu'en laboratoire, montrent la sensibilité du modèle à l'efficacité du système $\mathrm{PE}+$ Stabilisant ou encore à la sévérité des conditions d'exposition.

Le logiciel SIMUSTAB ${ }^{\circledR}$ permet également de calculer les durées maximales d'exposition d'un tube PE à proximité d'une canalisation de chauffage urbain, en prenant en compte, le cas échéant, l'histoire du tube lié au stockage (Fig. 6).

La température étant un facteur accélérateur des facteurs de ruine du PE tels que l'oxydation ou la fissuration en fluage - du fait de la thermodépendance des paramètres rhéologiques du matériau - il convient de cerner assez précisément les conditions pour lesquelles le tube risque de dépasser en permanence la température de $30{ }^{\circ} \mathrm{C}$, limite supérieure maximale tolérée au niveau des spécifications de Gaz de France.

Dans ce cadre, des calculs thermiques réalisés au moyen des logiciels FLUX-2D et de FLUX-3D permettent de calculer les distances inter concessionnaires à respecter en ce qui concerne la pose du tube PE nu et, au-delà, de calculer les caractéristiques « génériques » de l'isolant à intercaler pour que la température de peau du tube n'excède pas la limite spécifiée de $30{ }^{\circ} \mathrm{C}$ (Fig. 7).

\subsubsection{Phénomènes de fissuration liés à la présence d'entailles ou de défauts}

Le phénomène de rupture par fissuration lente est le mode de rupture à long terme reconnu du $\mathrm{PE}$ aux faibles contraintes, phénomène amplifié aux températures élevées (voisines de $80{ }^{\circ} \mathrm{C}$ ), du fait du comportement thermodépendant du matériau. Pour un tube exempt de défauts artificiels (ex. entailles, rayures, ...) il procède par désenchevêtrement progressif des macromolécules

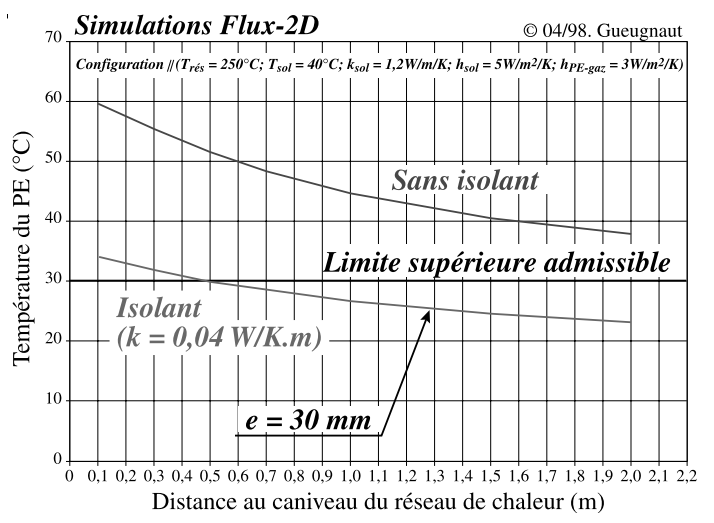

(a)

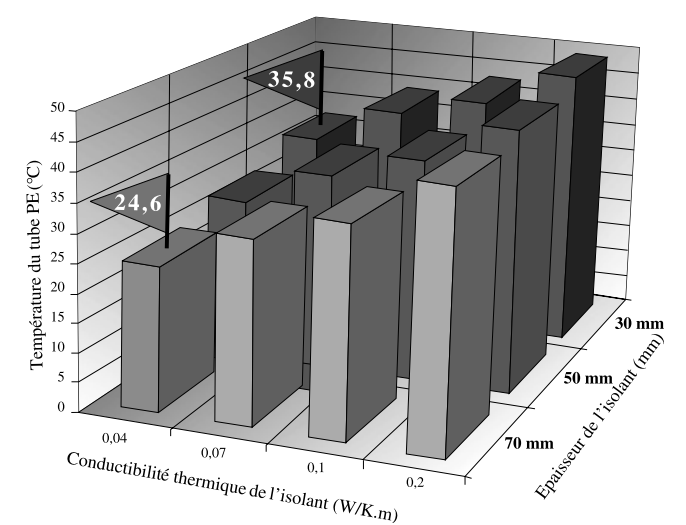

(b)

Fig. 7. Température de peau du tube PE en fonction de la distance au réseau de chaleur en l'absence et en présence d'un isolant concentrique (a). Température de peau du tube placé à $0,30 \mathrm{~m}$ du réseau de chaleur en fonction des caractéristiques de l'isolant concentrique (b). 


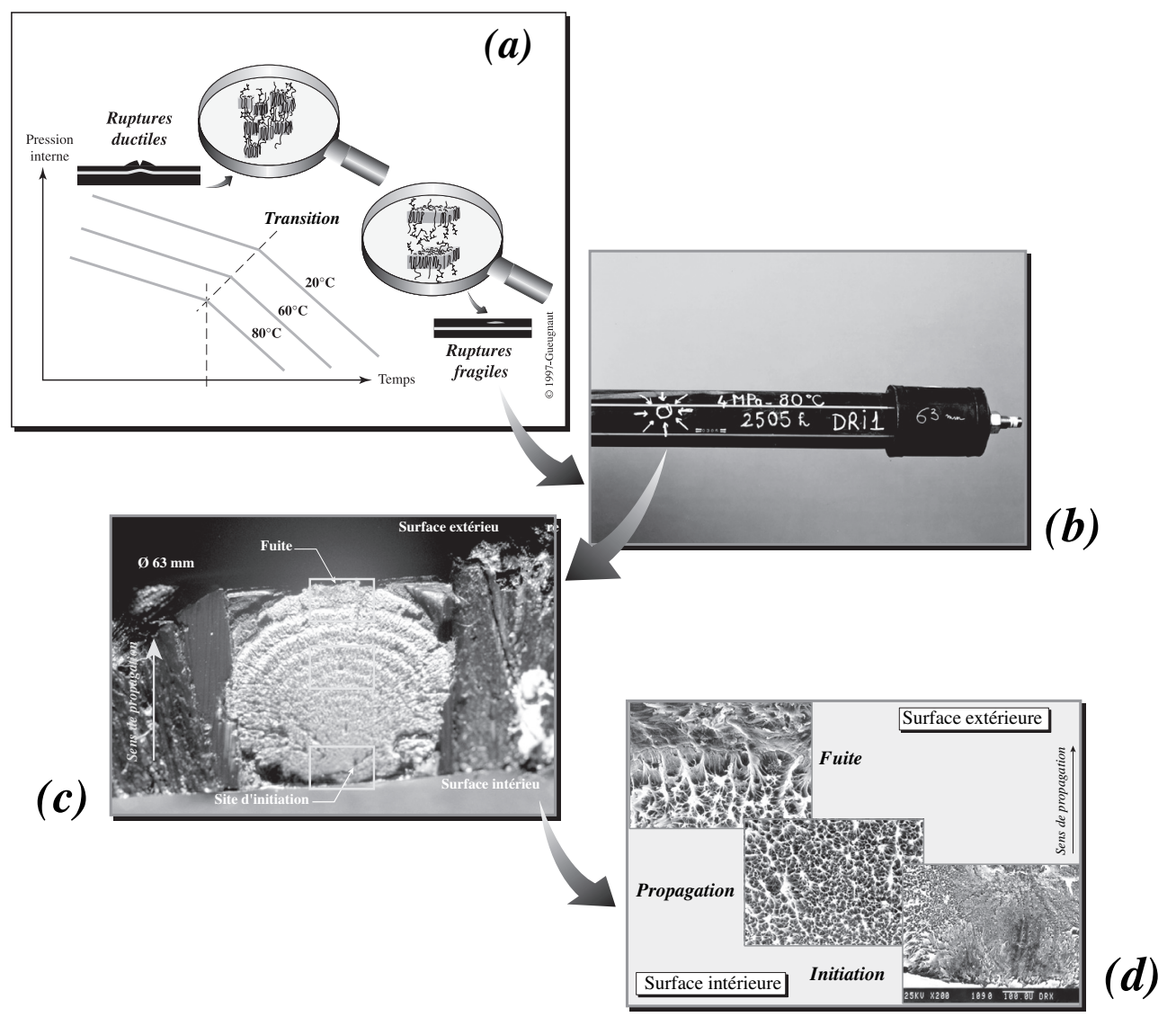

Fig. 8. Modes de rupture des tubes PE sous pression en fonction de la contrainte et de la température (a, b). Faciès de rupture fragile lente d'un tube soumis à $4 \mathrm{MPa} / 80{ }^{\circ} \mathrm{C}(\mathrm{c}, \mathrm{d})$.

démarrant sur un micro-défaut intrinsèque du matériau (micro-vide, résidu catalytique, ....), situé au voisinage de la surface intérieure du tube, la fissuration débouchant finalement en surface extérieure du tube (Fig. 8).

Dans le cas d'un tube présentant une entaille sur sa peau externe, le phénomène se trouve généralement inversé, du fait de la concentration des contraintes en pointe de l'entaille, toutes conditions égales par ailleurs.

L'initiation de fissure dépend alors de la géométrie de l'entaille pré-existante. Trois catégories de défauts ont ainsi été identifiées à partir de retours terrain.

Ces défauts, générés au moment du stockage ou de la pose, peuvent être schématisés classiquement par leur angle d'ouverture et par leur profondeur (Fig. 9).

Les entailles de type « V nocives vis-à-vis de la fissuration lente, sur la base d'essais sur éprouvettes de laboratoires circulaires entaillées - essais Full Notch Creep - une étude systématique a été réalisée en se focalisant sur ce type de défaut pour différents grades de résines polyéthylène couramment utilisés sur les réseaux de distribution de gaz.

La modélisation et la simulation numérique de ces conditions expérimentales permetent d'établir des cartes d'isovaleurs d'endommagement (déformation anélastique critique) dans un diagramme « angle d'ouvertureprofondeur $»$.

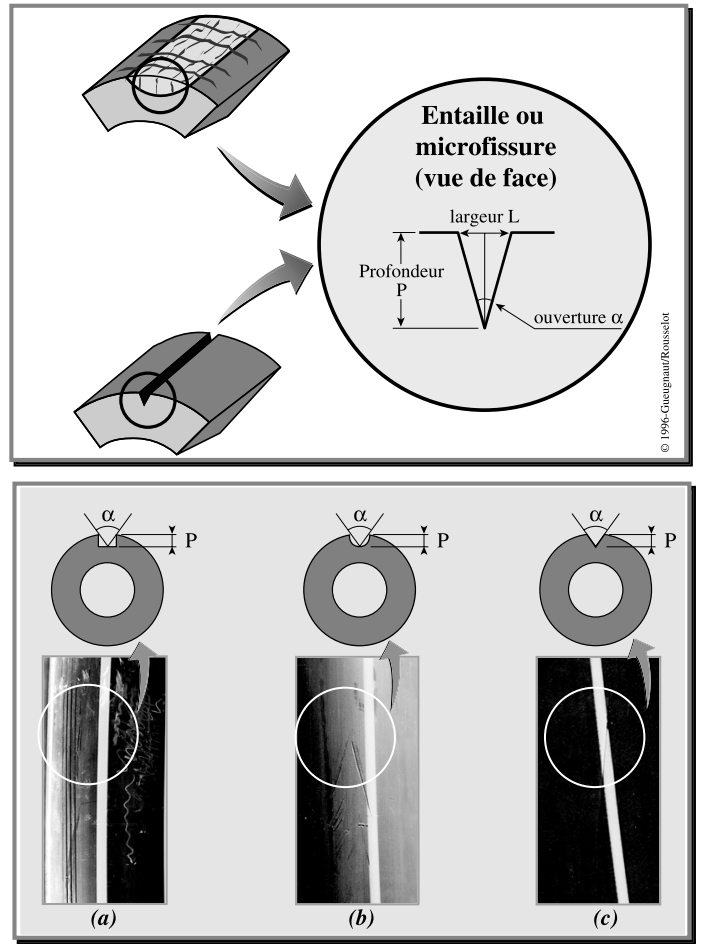

Fig. 9. Familles d'entailles caractéristiques rencontrées sur le terrain. 
(a)
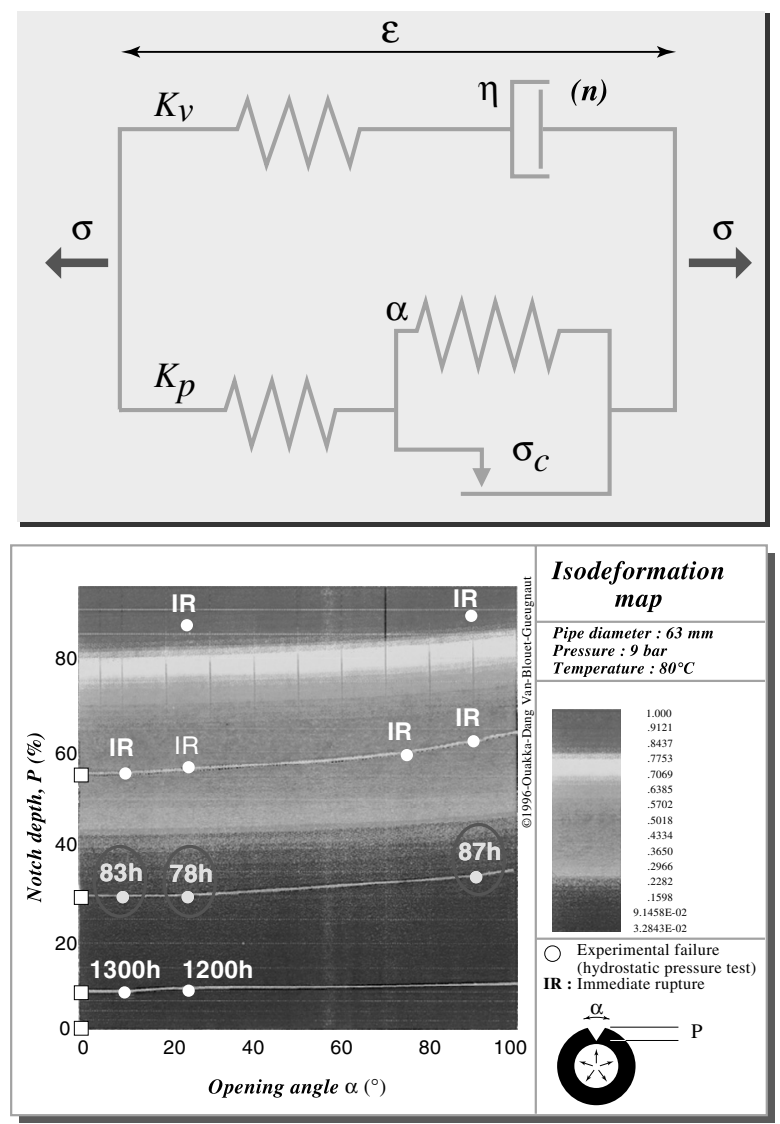

(b)

Fig. 10. Constitution du modèle rhéologique établi pour le PE (a) et carte de nocivité numérique d'un endommagement de type entaille en « $\mathrm{V} »$ avec superposition des résultats expérimentaux obtenus sur tubes entaillés (b).

La modélisation s'appuie sur un modèle rhéologique original à 6 paramètres prenant en compte les composantes élastique, visqueuse et plastique propres au PE, ainsi que sur une approche locale pour la description des phénomènes d'endommagement et de rupture.

Ces 6 paramètres, variables d'un grade PE à l'autre, constituent la « carte d'identité » rhéologique du matériau considéré [2].

Ces travaux constituent le prolongement de ceux initiés au début des années 90 par Gaz de France en collaboration avec le Laboratoire de Mécanique des Solides de l'École polytechnique [19-25].

En parallèle, des tubes entaillés avec différents couples « angle d'ouverture-profondeur » ont été mis sous pression constante et les temps de rupture correspondants relevés.

Les temps de rupture des tubes sous pression se répartissent de manière intéressante sur les isovaleurs d'endommagement ce qui autorise à établir une correspondance entre ces grandeurs (Fig. 10).

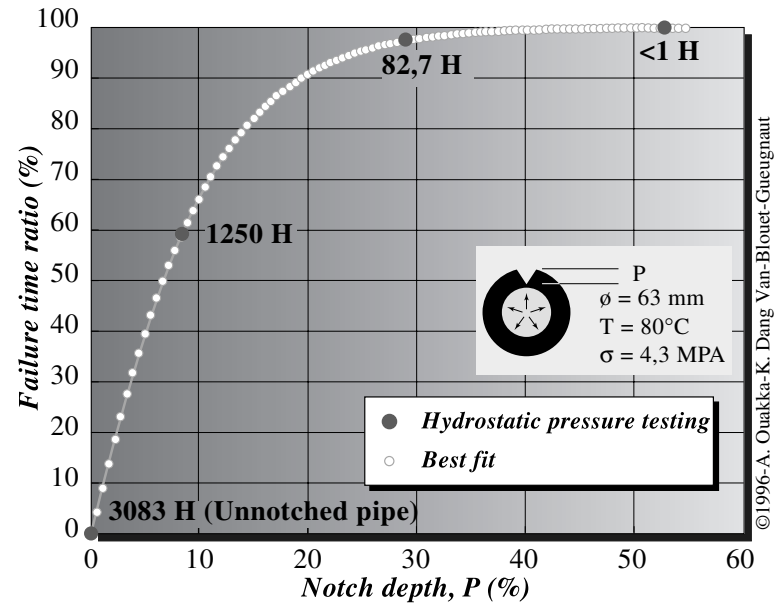

Fig. 11. Réduction du temps à la rupture en pression hydrostatique en fonction de la profondeur de l'entaille en $« \mathrm{~V} »$ pour des tubes $\mathrm{PE}$ endommagés.

Le report des valeurs des temps de rupture normalisés obtenus pour un angle d'ouverture voisin de zéro en fonction de la profondeur du défaut - exprimée en pourcentage de l'épaisseur de paroi du tube - permet d'évaluer la nocivité d'entailles de type « $\mathrm{V} »$ extrêmement pointues en fonction de leur profondeur, et d'en déduire une profondeur critique conservative (Fig. 11).

\subsection{Partie III : Évaluation et traitement des endommagements}

L'évaluation et la réparation des endommagements tant internes qu'externes aux canalisations PE fait appel à différentes méthodes intrusives ou externes, dont certaines sont actuellement en cours de développement par Gaz de France.

Actuellement est disponible une méthode de mesure quantitative des endommagements de type entaille. Cette méthode est basée sur la réalisation d'une empreinte négative de l'entaille et sur la mesure connexe des dimensions géométriques de celle-ci [25,26].

Le kit dit « de prise d'empreinte et de diagnostic » est composé de deux éléments séparés complémentaires à savoir : une malette contenant les pâtes nécessaires à la réalisation de la réplique de l'entaille, et un coffret contenant une loupe grossissante pourvue d'un réticule gradué et d'un rapporteur d'angle pour la mesure des dimensions de l'entaille (angle d'ouverture, profondeur).

Les composants utilisés pour la prise d'empreinte - dérivés de produits utilisés en dentisterie - ont été spécialement étudiés pour pouvoir être utilisés efficacement dans les conditions classiques de mise en œuvre $\mathrm{du} \mathrm{PE}$ sur chantier, soit classiquement entre $-5{ }^{\circ} \mathrm{C}$ et $+35{ }^{\circ} \mathrm{C}$, cette gamme d'utilisation n'étant nullement restrictive. 


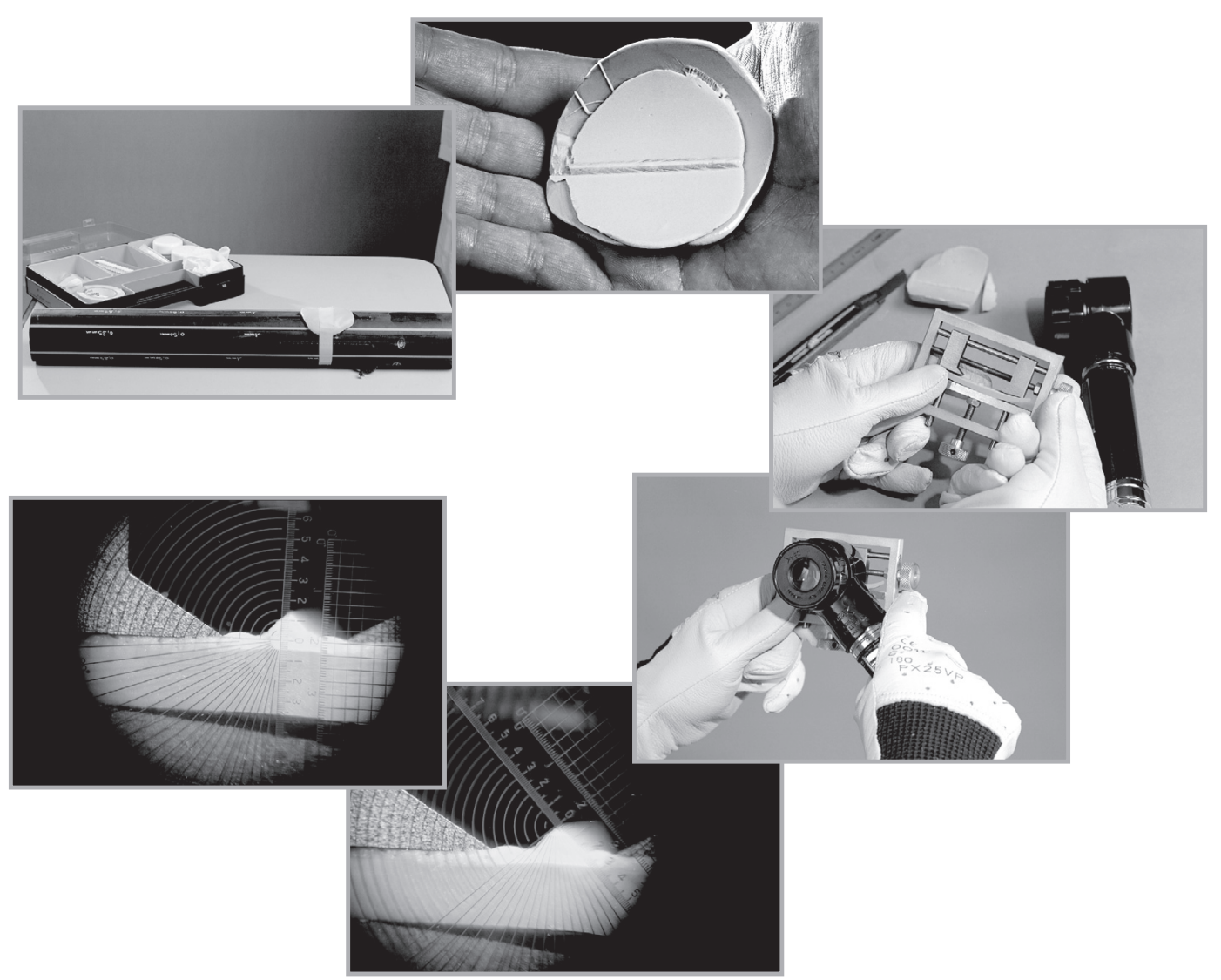

Fig. 12. Principe de la mesure des dimensions d'une entaille sur tube PE au moyen du kit de prise d'empreinte et de diagnostic.

Une fois la réplique réalisée, la mesure des dimensions de l'entaille est réalisée sur chantier au moyen du réticule gradué et du rapporteur d'angle intégrés à la loupe portative prévue à cet effet (Fig. 12).

La comparaison de la mesure obtenue, avec les profondeurs critiques (par diamètres de tube) indiquées dans la fiche jointe au kit de diagnostic, renseigne l'opérateur sur la nécessité ou non de déposer et de remplacer ou simplement de réparer le tronçon endommagé par les méthodes adéquates.

Une mesure contradictoire est prévue avant de remplir la fiche de prise d'empreinte qui pourra être jointe ultérieurement au dossier d'ouvrage le cas échéant (Fig. 13).

\subsection{Partie IV : Mots-clés}

Dans cette partie est donnée la signification des motsclés utilisés dans le guide, afin de donner au lecteur le vocabulaire commun pour une bonne compréhension des éléments du guide, et ce quelles que soient sa formation et ses connaissances initiales.

Des schémas permettent de compléter les définitions et ainsi de mieux appréhender les notions de base pour la compréhension de l'ouvrage comme par exemple l'endommagement, l'ovalisation, l'oxydation ou encore le comportement rhéologique (Fig. 14).
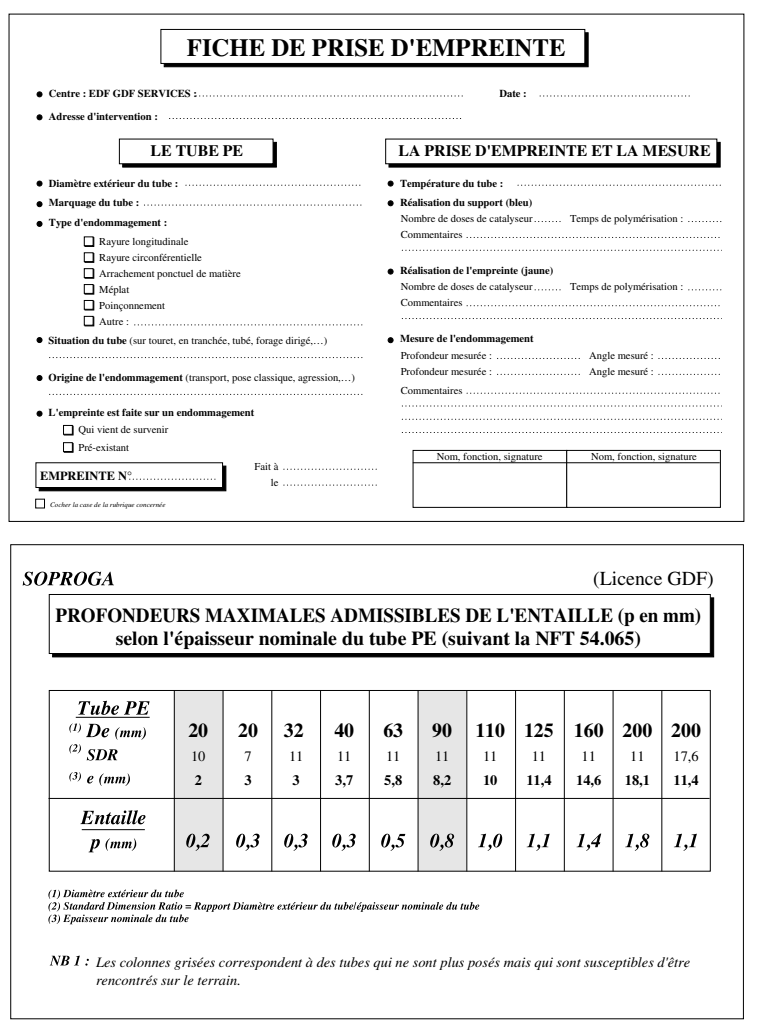

Fig. 13. Fiches de diagnostic de l'endommagement rattachées à la prise d'empreinte. 


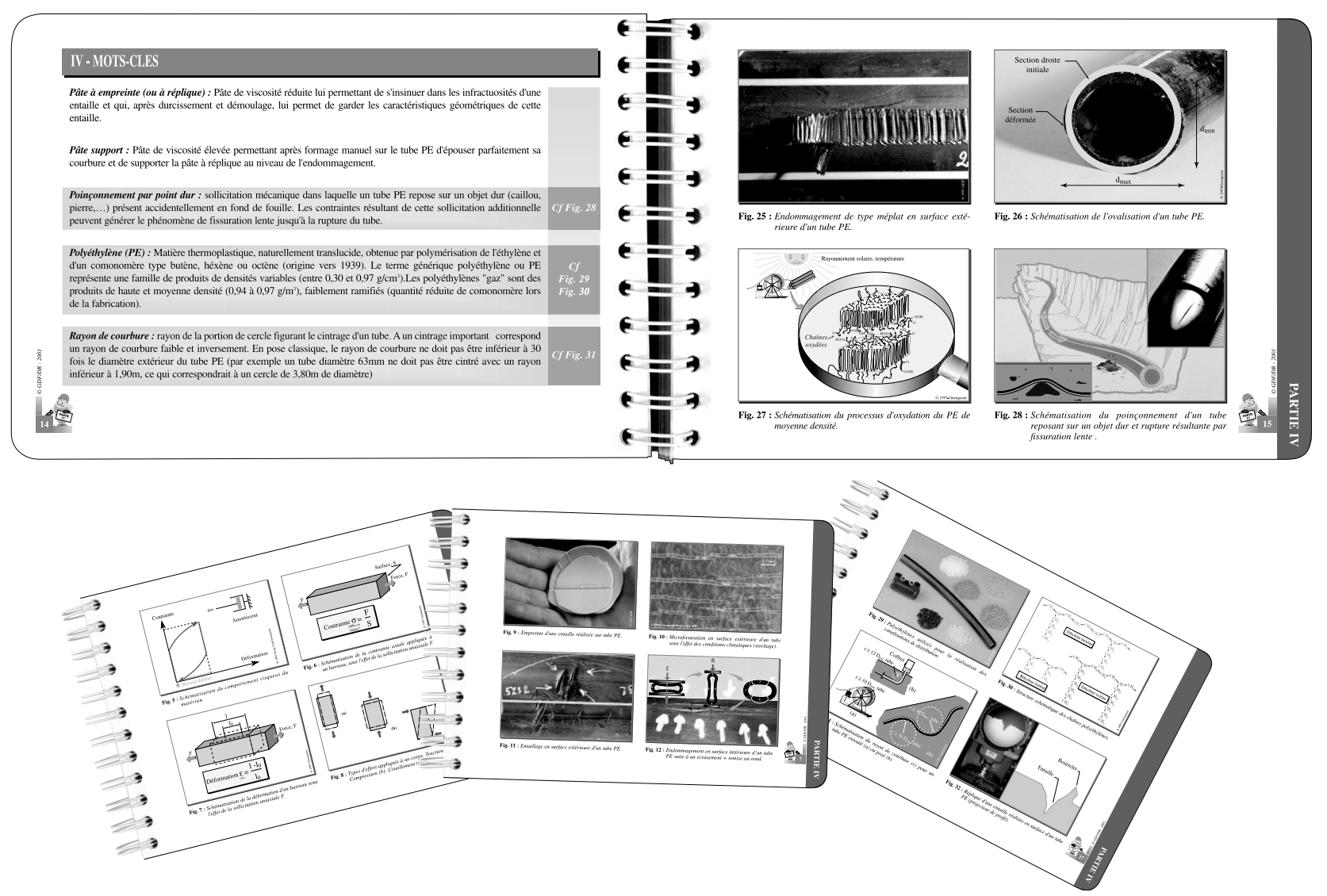

Fig. 14. Exemples de mots-clés et d'illustrations rattachées dans la partie IV du guide.

\section{Conclusion}

Le Guide de prévention, évaluation et traitement des endommagements constitue un vecteur d'information intéressant pour la formation des personnels chargés de mettre en œuvre les tubes et accessoires en polyéthylène pour la construction des réseaux de distribution au sens large (gaz, eau, ....).

En tant que tel, ce guide fait partie intégrante du «package $»$ des stages de formation dispensés par le Service de la formation professionnelle de Gaz de France pour la qualification des opérateurs PE.

Le concept volontairement modulaire de ce guide en fait un ouvrage vivant et évolutif qui se nourrit des résultats de recherche obtenus au fur et à mesure dans le domaine des canalisations PE.

Une version anglaise du guide a fait l'objet d'une première édition à l'automne 2002 [27,28].

\section{Références}

[1] D. Gueugnaut, D. Rousselot, International Plastic Pipe Symposium, Lake Buena Vista (Orlando, FLA), USA, October 20-23, 1997, p. 185

[2] D. Gueugnaut, P. Blouet, A. Ouâkka, K. Van Dang, International Gas Research Conference, San Diego (CA), USA, November 1-8, 1998, p. 35
[3] ISO 12176-4 : 2003(E), Plastics Pipes and FittingsEquipment for Fusion Jointing Polyethylene SystemsPart 4 : Traceability Coding, novembre 2003

[4] D. Dufour, Ch. Teygeman, La Traçabilité des Réseaux PE, $112^{\mathrm{e}}$ Congrès de l'Association Technique de l'Industrie du Gaz en France, Lille, France, 12-15 septembre 1995

[5] D. Gueugnaut, Ph. Deschamps, O. Cazenave, D. Dufour, 1998 International Gas Research Conference, San Diego (CA), USA, November 8-11, 1998, p. 57

[6] D. Gueugnaut, A. Darut, F. Dattolo, J.L. Toffart, Industrial Development of a Complete System for the Traceability of the Gas Distribution Networks Based on Bar Codes linked to the Polyethylene Pipes and Fittings, 22nd World Gas Conference, Tokyo, Japan, June 1-5, 2003

[7] D. Gueugnaut, A. Darut, F. Dattolo, Mise en place de la traçabilité. L'exemple des conduites de gaz en polyéthylène à Gaz de France, Gas Wasser und Abwasser, $83^{\text {e }}$ année, GWA 10/2003, SSIGE/VSA ed., Zürich, Octobre 2003, p. 725

[8] D. Dufour, J.C. Bergeron, J.C. Hugueny, Guide de l'Électrosoudage, Édition Gaz de France, SDIG Réf. 35.108.38, avril 1992

[9] D. Gueugnaut, A. Darut, J.C. Bergeron, Guide de Prévention, Évaluation et Traitement des Endommagements, ISBN 2-951-.6362-8, Édition Gaz de France $\mathrm{n}^{\circ}$ 2-9510636, CETAP Réf. 2TGZGPF1, dépôt légal septembre 2001 
[10] D. Gueugnaut, G. Lappai, P. Vautherin, D. Reveret, 12th Annual International Conference on Advances in the Stabilization and Controlled Degradation of Polymers, Luzern, Switzerland, May 21-23, 1990, p. 95

[11] D. Gueugnaut, Ch. Teygeman, G. Lappai, P. Vautherin, Étude sur l'évolution physico-chimique de tubes en polyéthylène soumis à des conditions de vieillissement climatique naturel, $9^{\text {es }}$ Journées d'Études sur le Vieillissement des Polymères, Bandol, France, 26-27 septembre 1991

[12] D. Gueugnaut, Ch. Teygeman, G. Lappai, P. Vautherin, International Gas Research Conference, Orlando (FLA), USA, November 16-19, 1992, p. 1006

[13] D. Gueugnaut, G. Lappai, P. Vautherin, D. Rousselot, 13th International Plastic Fuel Gas Pipe Symposium, San Antonio (TX), USA November 1-4, 1993, p. 252

[14] D. Gueugnaut, Ch. Teygeman, G. Lappai, P. Vautherin, Modélisation de l'évolution physico-chimique des tubes en polyéthylène utilisés pour la distribution du gaz. Part 1 : Stabilité résiduelle à l'oxydation, $11^{\text {es }}$ Journées d'Études sur le Vieillissement des Polymères, Bandol, France, 20-22 septembre 1995

[15] D. Gueugnaut, Ch. Teygeman, D. Rousselot, 17th International Conference on Advances in the Stabilization and Degradation of Polymers, Luzern, Switzerland, June 12-14, 1995, p. 369

[16] D. Gueugnaut, P. Blouet, A. Ouâkka, K. Van Dang, International Gas Research Conference, San Diego (Ca), USA, November 8-11, 1998, p. 269

[17] D. Gueugnaut, D. Rousselot, P. Schaaff, J.M. Quillevéré, D. Dufour, Colloque Annuel du Groupe Français des Polymères : Surfaces, Interfaces et Multimatériaux, P42, Mulhouse, France, 19-21 novembre 1996

[18] R. Pixa D. Gueugnaut, Méthodologie d'essai pour l'évaluation du comportement en pression des tubes en polyéthylène, $5^{\mathrm{e}}$ Colloque MECAMAT, Amnéville-LèsThermes, France, 6-9 avril 1992
[19] J. Kichenin, Comportement Thermomécanique du Polyéthylène. Application aux Structures Gazières, Thèse de l'École Polytechnique, janvier 1992

[20] A. Ouâkka, D. Gueugnaut, K. Van Dang, P. Blouet, $13^{\mathrm{e}}$ Congrès Français de Mécanique, Poitiers, France, 1-5 septembre 1997, p. 375

[21] P. Blouet, D. Gueugnaut, F. Marty, A. Ouâkka, European Conference on Macromolecular Physics : Surfaces and Interfaces in Polymers and Composites, Lausanne, Switzerland, June 1-6, 1997, p. 295

[22] A. Ouâkka, K. Van Dang, D. Gueugnaut, P. Blouet, 10th International Conference on Deformation, Yield and Fracture of Polymers, Cambridge, UK, April 7-10, 1997, p. 557

[23] A. Ouâkka, Rupture des Polymères : Approches numériques, Éléments Finis Polymères, Ed. J.C. Grandidier, C.G. Sell, 1997

[24] A. Ouâkka, K. Van Dang, D. Gueugnaut, P. Blouet, $3^{\text {e }}$ Congrès de Mécanique, Tome 1a, Tétouan, Maroc, 2225 avril 1997, p. 447

[25] D. Gueugnaut, P. Blouet, A. Ouâkka, K. Van Dang, International Gas Research Conference, San Diego (CA), USA, November 1-8, 1998, p. 269

[26] O. Cazenave, L. Maris, G. Seillant, La prise d'empreinte sur tubes PE, Congrès de l'Association Technique de l'Industrie du Gaz en France, Lille, France, 12-15 septembre 1995

[27] D. Gueugnaut, A. Darut, J.C. Bergeron, Prevention, Evaluation and Treatment of Damage to Polyethylene Piping, ISBN 2-951-06363-6, Édition Gaz de France $\mathrm{n}^{\circ}$ 29510636, CETAP Ref. 2TGZGPA1, Date of registration : December 2002

[28] D. Gueugnaut, F. Berthier, A. Darut, J.C. Bergeron, 17th International Plastic Fuel Gas Pipe Symposium, San Francisco (CA), USA, October 20-23, 2002, p. 189 